\title{
T-cell chronic lymphocytic leukaemia with pulmonary involvement and relapsing BOOP
}

\author{
E. Vaiman*, M. Odeh*, D. Attias**, Y. Ben-Arie***, A. Oliven*
}

\begin{abstract}
T-cell chronic lymphocytic leukaemia with pulmonary involvement and relapsing BOOP. E Vaiman, M Odeh, D Attias, Y Ben-Arie, A Oliven. C ERS Journals Ltd 1999.

ABSTRACT: We report on a case of T-cell chronic lymphocytic leukaemia involving the lung, with clinical, radiological and histological evidence of relapsing bronchiolitis obliterans-organizing pneumonia in a 70-yr-old female. Pulmonary disease was the major clinical manifestation of this chronic lymphocytic leukaemia. The first two episodes of the patient's pulmonary disorder resolved without treatment, and the third episode was treated with cytotoxic agents as part of the leukaemia treatment regimen. Two additional episodes of the pulmonary disorder occurred; both responded to prednisone.
\end{abstract}

Eur Respir J 1999; 14: 471-474.
Keywords: Bronchiolitis obliterans-organizing pneumonia

pulmonary involvement

T-cell chronic lymphocytic leukaemia

Received: December 41997

Accepted after revision March 31998
Pulmonary involvement in chronic lymphocytic leukaemia (CLL) is reported to be present at autopsy in up to $40 \%$ of cases [1]. However, clinical symptoms are uncommon, and significant pulmonary involvement as the major manifestation of CLL has rarely been reported [1-4]. All previously described cases were associated with B-cell-CLL (B-CLL) [1-4]. Only one of them was associated with bronchiolar involvement [2], and no case of bronchiolitis obliteransorganizing pneumonia (BOOP) associated with CLL has previously been reported. Here, we describe the case of a patient with a mixed pulmonary involvement of T-cell CLL (T-CLL) and relapsing BOOP.

\section{Case report}

A 70-yr-old female was admitted with a 3-month history of progressive dyspnoea, particularly on exertion, and a nonproductive cough without fever. In the previous few days, mild haemoptysis had also developed. Six months previously, she had been diagnosed as having CLL, on the basis of marked peripheral lymphocytosis (leukocyte count 16,000 cells $\cdot \mathrm{mm}^{-3}, 80 \%$ lymphocytes) with a characteristic peripheral blood smear. She had no history of asthma, and was a lifelong nonsmoker. Her other medical history was unremarkable, except for mild hypertension which was treated with hydrochlorothiazide. Chest radiography before admission was normal, and the patient was diagnosed by her family physician as having bronchitis. Treatment with antibiotics, cough mixtures and bronchodilators by means of inhalers was unsuccessful.

Physical examination showed tachypnoea (28 breaths. $\left.\min ^{-1}\right)$. Her blood pressure was $140 / 80 \mathrm{mmHg}$ and cardiac frequency 80 beats $\cdot \mathrm{min}^{-1}$; her body temperature and the results of the throat examination were normal. Lung examination revealed bilateral diffuse scanty wheezing and bibasilar inspiratory crackles. A few mildly enlarged lymph nodes were palpated bilaterally in the neck. The results of the heart and abdominal examination were normal with no hepatomegaly or splenomegaly.

The haemoglobin concentration was $12.8 \mathrm{~g} \cdot \mathrm{dL}^{-1}$, leukocyte count $18,000 \cdot \mathrm{mm}^{-3}(72 \%$ lymphocytes) with no eosinophilia, platelet count $20,7000 \cdot \mathrm{mm}^{-3}$ and lactate dehydrogenase activity $380 \mathrm{IU} \cdot \mathrm{L}^{-1}$ (normal $<240 \mathrm{IU} \cdot \mathrm{L}^{-1}$ ). The chest radiograph showed diffuse opacities involving both lungs (fig. 1a). A computerized tomography scan of the thorax revealed diffuse infiltrates in both lungs, without hilar or mediastinal lymphadenopathy. Arterial blood gas levels breathing room air were: arterial oxygen tension $\left(\mathrm{Pa}_{\mathrm{a}} \mathrm{O}_{2}\right) 7.0 \mathrm{kPa}(53 \mathrm{mmHg})$, arterial carbon dioxide tension $\left(\mathrm{Pa}_{\mathrm{a}}, \mathrm{CO}_{2}\right) 3.3 \mathrm{kPa}(25 \mathrm{mmHg})$, and $\mathrm{pH}$ 7.37. Pulmonary function tests showed: forced vital capacity (FVC) $1.45 \mathrm{~L}$ ( $64 \%$ of the predicted value), forced expiratory volume in one second (FEV1) $1.04 \mathrm{~L}$ (56\% pred), FEV1/FVC $72 \%$, forced mid-expiratory flow $1.24 \mathrm{~L} \cdot \mathrm{s}^{-1}$ (48\% pred), residual volume $2.20 \mathrm{~L}(118 \%$ pred) and total lung capacity $3.65 \mathrm{~L}$ ( $87 \%$ pred). There was no improvement following inhalation of a bronchodilator. Blood and sputum cultures were negative for bacteria and fungi. Sputum was also negative for malignant cells and Mycobacterium tuberculosis. Serological tests were negative for viral infection including human T-cell leukaemia virus-1 and human immunodeficiency virus. Echocardiography showed intact heart valves and normal ventricular systolic and diastolic functions. Cervical lymph node biopsy showed diffuse infiltration of small, well-differentiated T-lymphocytes. Microscopic examination of a bone marrow biopsy specimen showed extensive infiltration of the bone marrow by T-lymphocytes, most of them being CD4+ T-lymphocytes. There were also increased numbers of CD27 and CD3 T-lymphocytes in the bone marrow. Flow cytometry of bone marrow showed positive results for $\mathrm{CD} 2, \mathrm{CD} 3, \mathrm{CD} 5, \mathrm{CD} 7$ and $\mathrm{CD} 38$, and negative results for CD56, CD16 and CD 11b. Within a few days, spontaneous improvement in the patient's symptoms

*Dept of Internal Medicine B and **Institute of Haematology, Bnai Zion Medical Center, and ***Dept of Pathology, Rambam Medical Center, Technion Faculty of Medicine, Israel Institute of Technology, Haifa, Israel.

Correspondence: M. Odeh, Dept of Internal Medicine B, Bnai Zion Medical Center, P.O. Box 6477, Haifa 31063, Israel. Fax: 972 48371393 

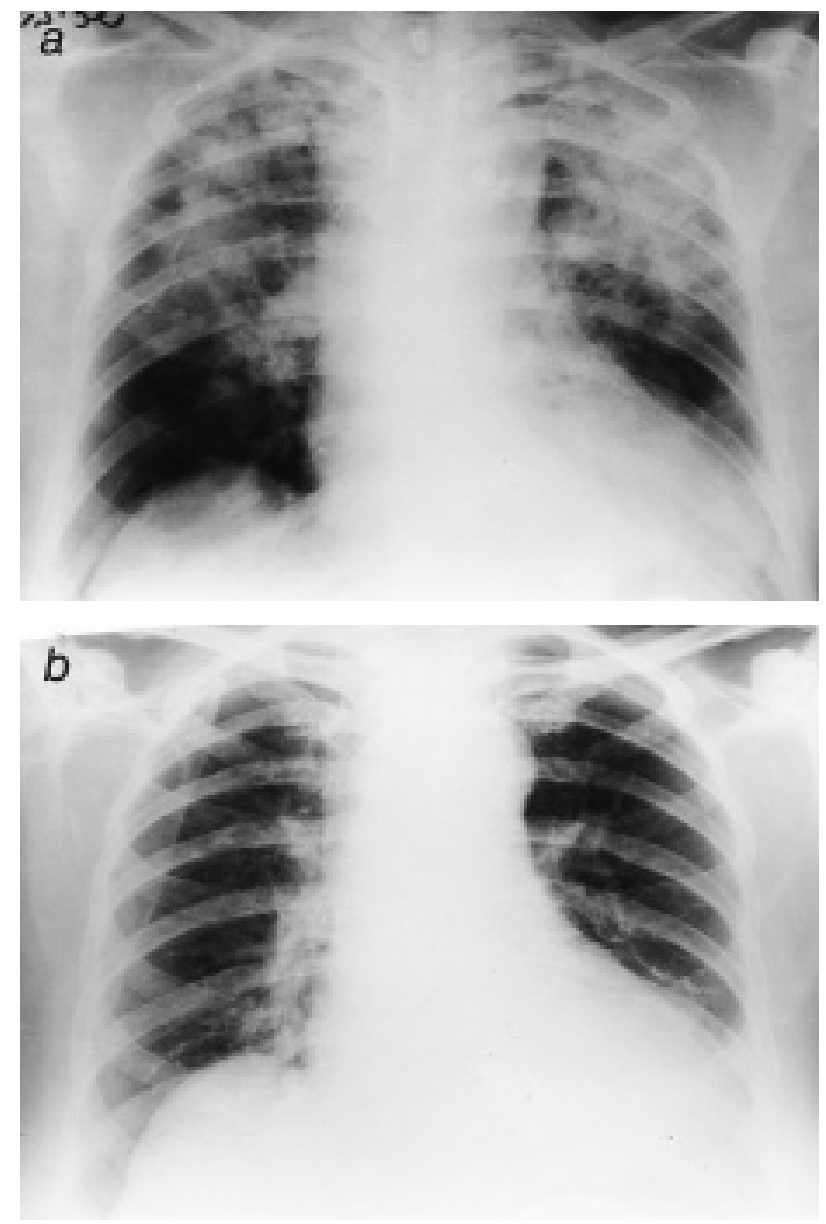

Fig. 1. - Chest radiographs showing: diffuse opacities involving both lungs; and normal lung fields after spontaneous amelioration of the patient's symptoms.

occurred. The $\mathrm{Pa}_{\mathrm{a}} \mathrm{O}_{2}$ increased to $11.7 \mathrm{kPa}(88 \mathrm{mmHg})$ and the lung opacities resolved completely on repeat chest radiography without any treatment (fig. 1b). The patient was discharged without a firm diagnosis regarding the pulmonary opacities, feeling well and with no cough or respiratory distress.

Three weeks later, the patient was readmitted for recurrent cough and respiratory distress. Physical examination revealed findings similar to those seen earlier, and the chest radiograph again demonstrated bilateral interstitial opacities. Fibreoptic bronchoscopy showed patent bronchi and no abnormality. Bronchial brushings and washings were negative for infection including acid-fast bacilli, fungi and Pneumocystis carinii. Bronchoalveolar lavage (BAL) was not performed because of significant respiratory distress during the bronchoscopy. Microscopic examination of a transbronchial lung biopsy specimen showed a mild interstitial mononuclear infiltrate, which was considered nondiagnostic. Once again, the patient's symptoms and lung opacities on chest radiography resolved spontaneously within a few days, and she was discharged without further therapy.

Two months later, the patient was readmitted for recurrent pulmonary disease. An open lung biopsy was performed. Histological examination of the lung biopsy specimen showed many foci of fibrosis in the air spaces. There were many masses and nodules made of elongated fibroblasts; some showed parallel orientation, embedded in a myxomatous palestaining stroma. Some fibrotic nodules were elongated, some roundish- and many serpiginous. The masses tended to form plugs and polyps in the alveolar ducts and spaces, and were covered by alveolar epithelial cells. In many alveolar spaces, there were collections of foamy lipid-containing macrophages (fig. 2). In addition, marked thickening of the alveolar septa was seen, mainly caused by lymphoplasmocytic infiltration. The lymphocytes were small and mature, and some of them were stained for mature T-cell markers (CD3, CD43, CD45RO). The rest of the lymphocytes were stained for mature B-cell markers (CD20, CD45RA, CDw75), and the plasma cells were polyclonal. In certain areas, there was marked infiltration by T-CLL. The infiltration was in the alveolar septa and spaces forming large masses (fig. 3). The malignant lymphocytes had small-to-medium-sized slightly irregular nuclei and there were also a few blasts. The leukaemic cells stained for mature T-cell markers. Immunostains for B-cells and terminal deoxynucleotidyl transferase were all negative. In a few areas, the blood vessels showed extensive leukaemic infiltration. All of the above findings were consistent with BOOP associated with focal infiltration by the T-CLL process in lung tissue. The biopsy specimen was negative for infection including with acid-fast bacilli and fungi. At that time, the leukocyte count was $69,300 \cdot \mathrm{mm}^{-3}$ (80\% lymphocytes), the majority of which were CD4+ T-lymphocytes.

The patient was treated with cytostatic drugs according to the (cyclophosphamide, mitozantrone, vincristine, prednisone (CMVP)) protocol. She underwent eight therapeutic courses with this protocol, each for 5 days with an interval of 3 weeks between the courses. Significant clinical improvement occurred within a few days, and on repeat chest radiography, the lung infiltrates had completely resolved within 2 weeks. Four months after the last therapeutic course with the CMVP protocol and while the patient was not being treated with chemotherapy or prednisone, an additional mild episode of the pulmonary infiltrate (presumably BOOP) associated with mild dyspnoea and cough recurred. This episode responded within a few days, to prednisone, which was continued for 3 months. Three months later, another similar mild episode developed, and again responded within a few days to prednisone, which was then continued for 6 months. Over a subsequent follow-up period of 6 months, no additional similar episodes were observed.

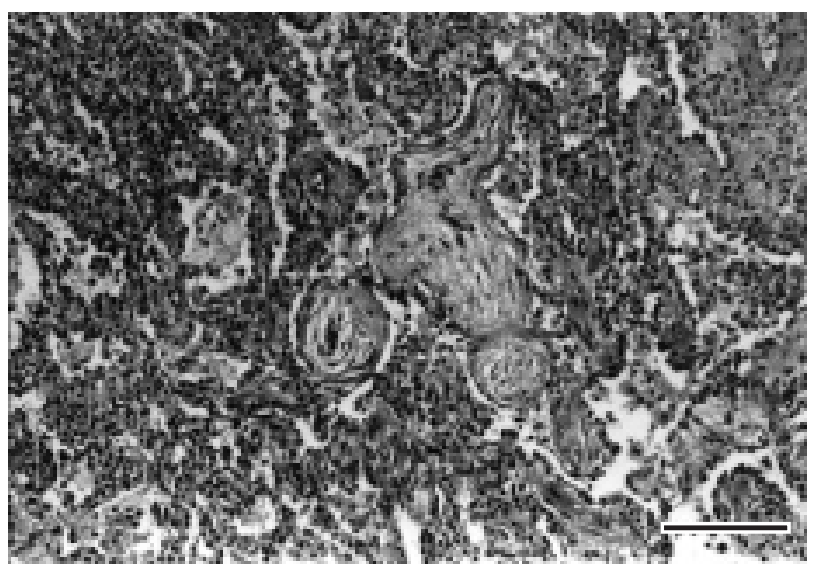

Fig. 2. - Photomicrograph showing intra-alveolar fibrotic plugs and focal alveolar accumulation of foamy macrophages (haematoxylin/eosin staining). (Internal scale bar=200 $\mu \mathrm{m}$.) 


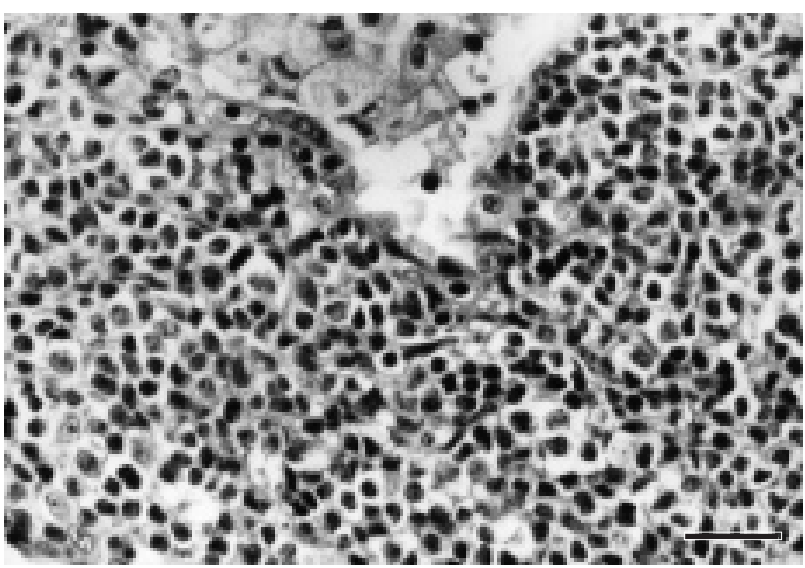

Fig. 3. - Photomicrograph showing heavy infiltration of leukaemic lymphocytes in the lung parenchyma. At the top there is a collection of intra-alveolar foamy cells (haematoxylin/eosin staining). (Internal scale bar $=50 \mu \mathrm{m}$.)

\section{Discussion}

T-CLL is very uncommon and accounts for $\sim 5 \%$ of all CLL cases and $<1 \%$ of all chronic lymphoproliferative diseases [5]. It is distinguished from B-CLL by an aggressive course, poor response to therapy and shorter survival. Lymphadenopathy and splenomegaly are uncommon [5]. The immunophenotype is that of mature T-cells, i.e. usually CD4+ [5], as in our patient.

The presence of pulmonary infiltrates in a patient with CLL poses a difficult diagnostic problem. It may be due to infection, haemorrhage, oedema or pulmonary infarction [6]. Our patient presented with progressive cough, dyspnoea and pulmonary infiltrates on her chest radiograph after a short period of asymptomatic CLL. She had no fever, and her pulmonary disease did not respond to antibiotics. Blood and sputum cultures and serological tests were negative for infectious agents, and echocardiography showed normal heart function. Also, there was no evidence of pulmonary haemorrhage, oedema, or infarction in the lung biopsy specimen.

The patient's initial pulmonary manifestations could have been due to direct pulmonary involvement by T-CLL, BOOP or both. The exact pathological diagnosis was only established after three similar episodes. Inasmuch as the first two episodes of our patient's respiratory symptoms and associated pulmonary infiltrates resolved spontaneously, and developed again despite the introduction of steroids and cytotoxic chemotherapy, we believe they were probably related to BOOP because T-CLL involvement is unlikely to vanish spontaneously.

BOOP associated with lung involvement of B- or T-CLL, as in the present case, has not previously been reported. Indeed in our case, the BOOP may have been elicited in some way by the lung involvement of T-CLL. However, we cannot rule out idiopathic BOOP, with a chance association with TCLL.

Direct pulmonary involvement of CLL is reported to be present at autopsy in up to $40 \%$ of cases [1]. However, CLL involvement in the form of significant clinical or radiographic manifestations is very uncommon, and pulmonary involvement as the initial manifestation of CLL has only rarely been reported [1-4]. Symptoms related to pulmonary involvement of B-CLL may appear 2-8 yrs after the diagnosis of CLL in patients with minimal peripheral blood smear abnormalities, as well as in patients in complete remission [1-4]. In the majority of patients with pulmonary CLL, however, the diagnosis is established via open lung biopsy, as in the present case $[1,2]$. Only in a few cases has the diagnosis been established by means of transbronchial lung biopsy or bronchoalveolar lavage $[1,3]$. Rarely, CLL may involve the pleura, causing pleural effusion [4]. Most of the cases previously reported as lung involvement of CLL were due to B-CLL. One single case of pleural involvement of TCLL and massive pleural effusion without lung involvement has been described [7].

BOOP is a clinical/pathological entity that may develop in response to or in association with a huge variety of pulmonary insults. Clinically, the idiopathic variety of BOOP may develop with subacute influenza-like symptoms, followed by cough and progressive dyspnoea [8]. Radiographically, the disease is characterized by multiple patchy alveolar infiltrates which do not respond well to antibiotics, hypoxaemia and a restrictive ventilatory defect [8]. The BAL fluid usually shows mixed cellularity, with the most consistent increase being seen in the percentage of lymphocytes, along with neutrophils and eosinophils in many cases. It also shows a significant decrease in the ratio of CD4+/CD8+ [9]. Histologically, it is characterized by patchy inflammatory changes in the bronchi and alveolar lumina and walls, an interstitial mononuclear cell infiltrate of variable density, obliteration of the airway lumen by intraluminar polyps of loose connective tissue containing inflammatory cells and fibroblasts and patchy areas of organizing pneumonia [10, 11]. Although the majority of BOOP cases are idiopathic, the syndrome has been described in association with many different settings, particularly collagen vascular disease [10, 12], and drugs [8]. It has also been described in the context of resolving infection, organizing diffuse alveolar damage, hypersensitivity pneumonitis, exposure to toxic fumes, organizing aspiration pneumonia, eosinophilic pneumonia, eosinophilic granuloma, Wegener's granulomatosis and around other lesions, including abscesses and tumours [13-15]. Three cases of BOOP have previously been reported in association with malignant lymphoma $[16,17]$, and a favourable response to steroids was observed in all of them. As stated above, BOOP has not previously been reported in association with CLL. It should be noted that BOOP has been observed in haematology patients in other settings, mainly following bone marrow transplantation [18-23].

The pathophysiology of BOOP is still unknown. However, its prognosis is generally good, and response to corticosteroids is excellent in most cases [8]. A grim prognosis has been observed in patients with collagen vascular disease [24], in whom a fulminant course with a fatal outcome has been reported for some patients [25-31].

In the present case, the patient presented with gradually worsening pulmonary disease with previously asymptomatic T-cell chronic lymphocytic leukaemia. A relapsing clinical and radiographic pattern of bronchiolitis obliterans-organizing pneumonia was observed during the course of her disease, and an open lung biopsy confirmed bronchiolitis obliterans-organizing pneumonia and pulmonary T-cell chronic lymphocytic leukaemia. Recurrent bronchiolitis obliterans-organizing pneumonia may be a pattern of response of the lung to a malignant lymphocytic infiltrate, especially T-CLL.

\section{References}

1. Rollins SD, Colby TV. Lung biopsy in chronic lymphocytic leukemia. Arch Pathol Lab Med 1988; 112: 607-611.

2. Palosaari DE, Colby TV. Bronchiolocentric chronic lymphocytic leukemia. Cancer 1986; 58: 1697-1698. 
3. Berkman N, Polliack A, Breuer R, Okon E, Kramer M. Pulmonary involvement as the major manifestation of chronic lymphocytic leukemia. Leuk Lymphoma 1992; 8: 495499.

4. Klatte EC, Yardley J, Smith EB. The pulmonary manifestations and complications of leukemia. Am J Roentgenol 1963; 89: 598-609.

5. Hoyer JD, Ross CW, Li C-Y, et al. True T-cell chronic lymphocytic leukemia: a morphological and immunophenotypic study of 25 cases. Blood 1995; 1163-1169.

6. Green RA, Nichols NJ. Pulmonary involvement in leukemia. Am Rev Tuberc Pulm Dis 1959; 80: 833-844.

7. Szalay F, Szathmari M, Paloczi K, Foldi J, Demeter J. Immunologic and molecular biologic characterisation of pleural involvement in a case of T-chronic lymphocytic leukemia. Chest 1994; 106: 1283-1285.

8. Costabel U, Guzman J, Teschler H. Bronchiolitis obliterans with organising pneumonia. outcome. Thorax 1995; 50 (Suppl 1): S59-S64.

9. Nagai S, Aung H, Tanaka S, et al. Bronchoalveolar lavage cell findings in patients with BOOP and related diseases. Chest 1992; 102 (Suppl. 1): 32S-37S.

10. Epler GR, Colby TV, McLoud TC, Carrington CB, Gaesler E. Bronchiolitis obliterans organising pneumonia. $N$ Engl $J$ Med 1985; 312: 152-158.

11. Colby TV. Pathologic aspects of broncholitis obliterans organizing pneumonia. Chest 1992; 102: 385-435.

12. Gammon RB, Bridges TA, Al-Nezir H, Alexander CB, Kennedy JI Jr. Bronchiolitis obliterans organizing pneumonia associated with systemic lupus erythematosus. Chest 1992; 102: 1171-1174.

13. King TE. BOOP: an important cause of migratory pulmonary infiltrates? Eur Respir J 1995; 8: 193-195.

14. Yousem SA. Bronchocentric injury in Wegener's granulomatosis. Hum Pathol 1991; 22: 535-540.

15. Uner AH, Rozum-Slota B, Katzenstein A-L. A Bronchiolitis obliterans-organizing pneumonia (BOOP)-like variant of Wegener's granulomatosis. A clinicopathologic study of 16 cases. Am J Surg Pathol 1996; 20: 794-801.

16. Romero S, Martin C, Massuti B, Asanda I, Hermander L. Malignant lymphoma in a patient with relapsing obliterans organizing pneumonia. Chest 1992; 102: 1895-1897.

17. Gosink BB, Friedman PJ, Liebow AA. Bronchiolitis obliterans: roentgenologic-pathologic correlation. Am J Roentgenol 1973; 117: 816-832.

18. Tenholder MF, Becker GL, Cervoni MI. The myelodysplastic syndrome and bronchiolitis obliterans. Ann Intern Med 1990; 112: 714-715.

19. Stemmelin GR, Bernaciak J, Casas JG. Bronchiolitis with leukemia. Ann Intern Med 1991; 114: 912-913.

20. Forsyth CJ, Cremer PD, Torzillo P, Iland HJ, Young GAR. Thalidomide responsive chronic pulmonary GVHD. Bone Marrow Transplant 1996; 17: 291-293.

21. Kanada Y, Takahashi T, Imai Y, et al. Bronchiolitis obliterans organizing pneumonia after syngeneic bone marrow transplantation for acute lymphoblastic leukemia. Bone Marrow Transplant 1997; 19: 1251-1253.

22. Lohr RH, Boland BJ, Douglas WW, et al. Organizing pneumonia. Features and prognosis of cryptogenic, secondary, and focal variants. Arch Intern Med 1997; 157: 13231329.

23. Kleinau I, Perezcanto A, Schmid HJ, et al. Bronchiolitis obliterans organizing pneumonia and chronic graft-versus-host disease in a child after allogeneic bone marrow transplantation. Bone Marrow Transplant 1997; 19: 841-844.

24. Presas JL, Piriz J, Serra SL, et al. Bronchiolitis obliterans organizing pneumonia associated with idiopathic thrombocytopenic purpura. Medicina (B Aires) 1998; 58: 58-60.

25. Guerry-Force ML, Muller NL, Wright JL, et al. A compaparision of bronchiolitis obliterans with organizing pneumonia, usual interstitial pneumonia and small airways disease. Am Rev Respir Dis 1987; 135: 705-712.

26. Cohen AJ, King TE, Downey GP. Rapidly progressive bronchiolitis obliterans with organizing pneumonia. Am J Respir Crit Care Med 1994; 149: 1670-1675.

27. Bartter T, Irwin RS, Nash G, Balikian JP, Hollingsworth HH. Idiopathic bronchiolitis obliterans organizing pneumonia with peripheral infiltrates on chest roentgenogram. Arch Intern Med 1989; 149: 273-279.

28. Usui Y, Kimula Y, Miura H, et al. A case of bronchiolitis obliterans organizing pneumonia associated with primary Sjögren's syndrome who died of superimposed diffuse alveolar damage. Respiration 1992; 59: 122-124.

29. Clawson K, Oddis CV. Adult respiratory distress syndrome in polymyositis patients with the anti-Jo-1 antibody. Arthr Rheum 1995; 38: 1519-1523.

30. Tasaka S, Kanazawa M, Kawai C, et al. Fatal diffuse alveolar damage from bird fancier's lung. Respiration 1997; 64: 307309.

31. Romero S, Hernandez L, Gil J, Aranda I, Martin C, SanchezPaya J. Organizing pneumonia in textile printing workers. A clinical description. Eur Respir J 1998; 11: 265-271. 